\title{
PROJECTIONS OF CANTOR SETS, SIMPLE CLOSED CURVES, AND SPHERES IN $E^{3}$
}

\author{
MARK D. MEYERSON*
}

1. Introduction. The main theorem in this paper is that in $E^{3}$ there is a tame 2-sphere with a Cantor subset such that all projections of the two sets are the same. There are several interesting related results. One corollary is that every tame knot class contains a knot which cannot be linked by a straight line. Compare this to the well-known result that every knot can be linked by a polygonal loop. Another implication solves a problem posed to the author by R. H. Bing, namely there does exist a simple closed curve in $E^{3}$ with Cantor subset such that all projections of the two sets are the same. The chief previous result in the area of this paper is due to $K$. Borsuk who showed, [4], that there is an arc whose projection in every plane has interior points.

2. Terminology. We say a $k$-sphere (or Cantor set) in $E^{n}$ is tame if there is a homeomorphism of $E^{n}$ onto itself which carries the $k$-sphere (or Cantor set) to a polyhedral $k$-sphere (or standard Cantor set - a subset of a line). A 2-sphere in $E^{3}$ is tame iff there is a homeomorphism of $E^{3}$ onto itself carrying the 2-sphere onto the standard unit sphere (by [1]). A Cantor set $C$ in $E^{3}$ is tame iff for each $\epsilon>0, C$ can be covered by a finite collection of mutually exclusive open 3cells of diameter less than $\epsilon$ (by [3, p. 435]). A knot and a straight line in $E^{3}$ will be called linked if they are disjoint and there is no isotopy of $E^{3}$ pushing the knot and line to different sides of some plane (this is stronger than some other possible definitions). A projection is defined geometrically as follows. For $X \subset E^{3}, M$ a plane, the projection of $X$ onto $M$ consists of those points $m$ of $M$ such that the line through $m$ perpendicular to $M$ meets $X$.

3. Main results.

THEOREM. In $E^{3}$ there exists a tame 2-sphere with a Cantor subset such that all projections of the two sets are the same.

We postpone the proof of the theorem to the last section. First we summarize the idea of the proof and discuss some corollaries.

Received by the editors on June 17, 1974, and in revised form on September 24, 1974.

*The author is an NSF fellow.

Copyright $\odot 1976$ Rocky Mountain Mathematics Consortium 
Idea of Proof. Consider $S_{0}$, a P.L. 2-sphere, inside $\Delta_{0}$, a 3-simplex. Any line meeting $S_{0}$ meets Int $\Delta_{0}$. Triangulate $\Delta_{0}$ into $\left\{\Delta_{1, i}\right\}$ and push out spikes on $S_{0}$ to get $S_{1}$ a P.L. 2-sphere which meets each Int $\Delta_{1, i}$. We can alter each $\Delta_{1, i}$ slightly so that any line meeting $S_{1}$ meets some Int $\Delta_{1, i}$. We shrink each $\Delta_{1, i}$ slightly so this still holds and now $\left\{\Delta_{1, i}\right\}$ are pairwise disjoint. Continuing we get a sequence of P.L. 2-spheres $\left\{S_{n}\right\}$ and P.L. 3-cells $\left\{\Delta_{n, i}\right\}$, so that any line meeting $S_{n}$ meets some Int $\Delta_{n, i}$ and $S_{n+1}$ is "pushed out" from $S_{n}$ in $\bigcup_{i}$ Int $\Delta_{n, i}$. This can be done so that $C=\bigcap_{n} \bigcup_{i} \Delta_{n, i}$ is a Cantor set; so that $S_{n}$ approaches a tame 2 -sphere $S$ (in a precise sense), $C \subset S$, and any line meeting $\mathrm{S}$ meets $C$.

Conollary 1. There exists a simple closed curve in $E^{3}$ which cannot be linked by a straight line.

Proof. For $H: S^{2} \rightarrow S, S$ as in the theorem, $H$ a homeomorphism, construct $K^{\prime} \subset S^{2}$ a simple closed curve passing through the Cantor set $H^{-1}(C)$. Then $K=H\left(K^{\prime}\right)$ is the desired curve. For if $l$ is a line disjoint from $K$ then $l$ is disjoint from $S$ by the theorem. By the tameness of $S$, we can shrink $S$, and hence $K$, by an isotopy, toward a point inside $S$. This shows that $l$ and $K$ are not linked.

Conollary 2. (Bing's problem) There exists a simple closed curve in $E^{3}$ with Cantor subset such that all projections of the two sets are the same.

Proof. Use $K$ as in Corollary $1, C \subset K \subset S$. Since all projections of $C$ and $S$ are the same, all projections of $C$ and $K$ will be the same.

Corollary 3. Furthermore, in Corollaries 1 and 2 , we can take $K$ equivalent to any tame knot.

Proof. A small arc of $K$ which misses $C$ can be pulled inside $S$ and knotted as desired there (the original $K$ is unknotted since it lies in a tame 2-sphere). It may be easier to see that this can be done by performing the knotting on $K^{\prime}$ inside $S^{2}$ and then using $H$, where $H$ now has been extended to all of $E^{3}$. Let $\pi$ be an arbitrary projection. Since $\pi C=\pi S=\pi(S \cup$ interior $S)$ and $C \subset K \subset S \cup$ interior $S$, we immediately have $\pi C=\pi K=\pi S$. It is now clear that this knotted $K$ is as desired.

Corollary 4. Consider $\mathrm{S}^{2}$ as a transparent material with an opaque equator loop. Then there exists an ambient isotopy $H$ of $E^{3}$ so that

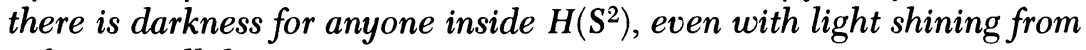
infinity in all directions. 
Proof. In the proof of the theorem, the homeomorphism $H$ is actually the last stage of an isotopy. The arguments of the proof are easily adapted so that any line from infinity meets $C$ before meeting $S-C$. In particular, in the proof of Lemma 1, if we use a mesh of $\epsilon / 6$ instead of $\epsilon / 4$ we see that a line from inininity meets $P$ in $(R T)$ before intersecting $P^{\epsilon}$, length $(\mathrm{RT})=\min (\epsilon, \sigma)$. It follows as in the proof of Lemma 1 that the line will meet $\bigcup_{n}\left[\left(\Delta^{\prime}{ }_{n}\right)^{\gamma}\right]$ before $P \epsilon$, and the remainder of the proof is basically unchanged. Finally, it is wellknown that there is an ambient isotopy taking $S^{2}$ to $S^{2}$ and any Cantor set in $S^{2}$ to a Cantor set on the equator, e.g., by methods similar to [2, pp. 307-310].

Note. These results can be intuitively stated in many ways: e.g., we could speak of shadows or lines of sight instead of projections.

4. Preliminaries. Everything is done in $E^{3}$.

Definition. For $\epsilon>0, \quad X \subset E^{3}, \quad X^{\epsilon}=\{x \in X: d(x, \partial X) \geqq \epsilon\}$. Equivalently, $\quad X^{\epsilon}=\left\{x \in X: B_{\epsilon}(x) \subset X, \quad\right.$ where $B_{\epsilon}(x)=\{y:|x-y|$ $<\epsilon\}$. Note that $X^{\epsilon}$ is a closed subset of Int $X$.

Lemma 1. Given a polyhedron $P$, with triangulation into 3-simplices $\left\{\Delta_{i}\right\}_{i=1}^{n}$ and $\delta, \epsilon>0$, there exists an isotopy $F$ such that:

(i) $F$ is fixed on $\partial P$.

(ii) F moves points by at most $\delta$.

(iii) $\Delta^{\prime}{ }_{i}$, the final image of $\Delta_{i}$ under $F$, is a polyhedron.

(iv) There is a $\gamma>0$, such that if a line meets $P^{\epsilon}$, then it meets $\bigcup_{i}\left[\left(\Delta^{\prime}{ }_{i}\right)^{\gamma}\right]$.

Furthermore, if $K$ is a compact subset of $P$ which misses the 1skeleton of $\left\{\Delta_{i}\right\}$, then we can assume that $F$ is fixed on $K$.

Proof. Let $\sigma$ be the distance from $K$ to the 1-skeleton of $\left\{\Delta_{i}\right\}$. Subdivide $\left\{\Delta_{i}\right\}$ into $\left\{\Gamma_{j}\right\}$ with mesh less than $\epsilon / 4$ and $\sigma / 7$. We can assume that if three vertices of $\left\{\Gamma_{j}\right\}$ determines a 2-face and also lie in $\partial P$, then the 2 -face lies in $\partial P$ (say by constructing $\left\{\Gamma_{j}\right\}$ using iterated barycentric subdivision). Let $E$ be the set of vertices of $\left\{\Gamma_{j}\right\}$ and let $K^{\prime}$ be the closed $\sigma / 7$ neighborhood of $K$. Then $E$ can be shifted slightly to $E^{\prime}$ so that:

(i) Vertices lying in $K^{\prime} \cup \partial P$ don't move.

(ii) By linear extension, the small shift $E \rightarrow E^{\prime}$ defines an isotopy $F$ taking $\Gamma_{j} \rightarrow \Gamma^{\prime}{ }_{j}$. We can assume $E^{\prime}$ is close enough to $E$ so that points in (Int $P)-K^{\prime}$ remain in $(\operatorname{Int} P)-K^{\prime}, F$ is an isotopy moving points by at most $\delta$, and the mesh of $\left\{\Gamma^{\prime}{ }_{j}\right\}$ is less than $\epsilon / 4$ and $\sigma / 7$ (A small distortion of a triangulation remains a triangulation by $[6$, p. 370] ). 
(iii) If $E_{1}, E_{2}$, and $E_{3}$ are disjoint unordered triples from $E-K^{\prime}$, such that at least one point of each $E_{i}$ is not in $\partial P$, then the 3 planes they determine (i.e., $E_{1}$ determines one plane, etc.) have exactly one point in common.

$F$ induces isotopies $\Delta_{i} \rightarrow \Delta^{\prime}{ }_{i} . \Delta^{\prime}{ }_{i}$ is a polyhedron since it is the union of certain $\Gamma^{\prime}{ }_{j}$.

Suppose 1 is a line meeting $P^{\epsilon}$. Then 1 contains an open segment $(R S) \subset$ Int $P$ meeting $P^{\epsilon}$ and with $R$ and $S$ in $\partial P$.

If $R$ is not in the 1 -skeleton of $\left\{\Delta_{i}\right\}$, then $R$ is a positive distance from all but one $\Delta_{i}$. It then follows, since $F$ is fixed on $\partial P$, that $R$ is a positive distance from all but one $\Delta^{\prime}{ }_{i}$, and hence 1 meets some Int $\Delta^{\prime}{ }_{i}$.

If $R$ is in the 1-skeleton of $\left\{\Delta_{i}\right\}$, then there is a segment $(R T) \subset$ $(R S)$ so that $(R T) \cap K=\varnothing$ and length $(R T)=\min (2 \epsilon, \sigma) . \quad(R T)$ must meet in segments at least 3 disjoint $\Gamma^{\prime}{ }_{j}$ none of which meet $K^{\prime}$. If $(R T)$ met all three only in 2 -faces, the faces would lie in Int $P$, which leads to a contradiction by the general position assumption of (iii) above. So $(R T)$ meets some Int $\Gamma^{\prime}{ }_{j}$. Hence 1 meets some Int $\Delta^{\prime}{ }_{i}$. (The above argument bears some resemblance to that of Borsuk in [4] ).

On the compact space of lines meeting $P^{\epsilon}$ consider the continuous positive function which assigns to a line 1 the largest $\gamma>0$ such that for some $x \in 1$ and some $n, B_{\gamma}(x) \subset \Delta^{\prime}{ }_{n}$ (as done in [4, p. 274]). This function achieves a minimum $\gamma>0$, and so if 1 meets $P^{\epsilon}$ it meets $\bigcup_{n}\left[\left(\Delta^{\prime}{ }_{n}\right)^{\gamma}\right]$. This completes the proof.

LEMMA 2. If $X \subset E^{3}, h_{1}: X \rightarrow E^{3}, h_{n}$ : range $\left(h_{n-1}\right) \rightarrow E^{3}$, for $n \geqq 1$ $h_{n}$ is a continuous function moving points by less than $\epsilon_{n}$ and $\sum \epsilon_{n}<\infty$, then $h_{n} \circ h_{n-1} \circ \cdots \circ h_{1}$ converges (uniformly) to a continuous function $h$. If $X$ is closed, then range $(h)=\left\{x \in E^{3}\right.$ : given $\epsilon>0$, there exists $N$ such that for $n>N$, dist $\left(x\right.$, range $\left.\left.\left(h_{n}\right)\right)<\epsilon\right\}$. (Equivalently, range $(h)=$ lim inf range $\left(h_{n}\right)$.)

Proof. Let $H_{n}=h_{n} \circ h_{n-1} \circ \cdots \circ h_{1}: X \rightarrow E^{3}$. It is immediate that $H_{n}$ converges to a continuous $h$ which moves points by less than $\sum \epsilon_{n}\left(\left\{H_{n}\right\}\right.$ forms a Cauchy sequence in the complete space of continuous functions from $X$ to $\left.E^{3}\right)$. We let $R(g)=\operatorname{range}(g)$.

Clearly $R(h) \subset \lim \inf R\left(h_{n}\right)$. Let $x \in \lim \inf R\left(h_{n}\right)$. Then there exists $\left\{x_{n}\right\} \subset X$ such that $H_{n}\left(x_{n}\right) \rightarrow x .\left\{H_{n}\left(x_{n}\right)\right\}$ is a bounded sequence and each $H_{n}$ moves points by less than $\sum \epsilon_{i}$, so $\left\{x_{n}\right\}$ is a bounded sequence. Hence there exists subsequence $x_{n_{j}} \rightarrow x_{0}$ for some $x_{0}$ in $X$ (closed).

We show that $H_{n_{j}}\left(x_{0}\right) \rightarrow x$. Fix $a>0$ arbitrarily. There exists $N$, such that for $m>N, h_{m} \circ \cdots \circ h_{N+1}$ moves points by less than $a / 3$. Let 
$B$ be a compact set containing $\left\{x_{n}\right\}$. Then there is a $b>0$, such that for $d(x, y)<b$ and $x, y \in B$, we have $d\left(H_{N}(x), H_{N}(y)\right)<a / 3$. And so, for $x, y \in B, d(x, y)<b$, we have $d\left(H_{m}(x), H_{m}(y)\right)<a$. Since $x_{n_{j}} \rightarrow x_{0}$ in $B$ and $a$ was arbitrary, $d\left(H_{n_{j}}\left(x_{n_{j}}\right), H_{n_{j}}\left(x_{0}\right)\right) \rightarrow 0$. But $H_{n_{j}}\left(x_{n_{j}}\right) \rightarrow x$, so $H_{n_{j}}\left(x_{0}\right) \rightarrow x$.

Since $H_{n}\left(x_{0}\right) \rightarrow h\left(x_{0}\right), x=h\left(x_{0}\right) \in R(h)$ and we are done.

LEMMA 3 (technical lemma). Given a square in a horizontal plane, consider a right cylinder with the square as base and height at least double the length of a side of the square (see figure $1 a$ ). Then there exists a homeomorphism $h$ of the plane onto the plane together with the surface of the cylinder, minus the original square's interior, such that:

(i) $h$ is the identity outside the square.

(ii) $h$ is a local dilation (i.e., any point in the domain of $h$ has a neighborhood so that for $x$ and $y$ points in the neighborhood, $d(h(x)$, $h(y)) \geqq d(x, y))$.

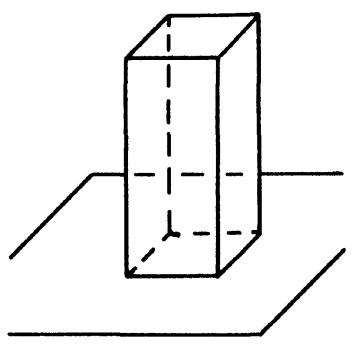

(a)

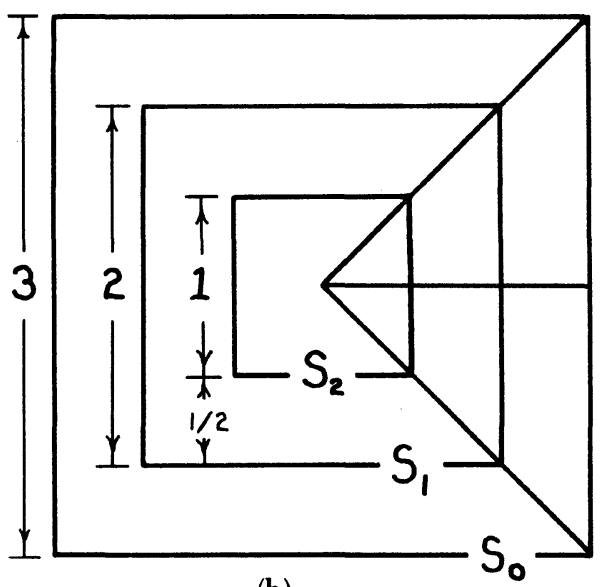

(b)

Figure 1

(Note: Although the truth of this lemma may seem obvious, it is trickier than might be first supposed. To give some indication of the possible problems we note the following. There is no local dilation of an isoceles right triangle onto the square, two of whose sides are the legs of the triangle, such that the dilation map is the identity on the 
two legs. Proof: Suppose there were such a map. It would not decrease the length of any arc. But the pre-image of the hypotenuse decreases in length, a contradiction. As another example, we note that if the cylinder in the lemma were not a right cylinder, a homeomorphism of the type used here would fail to be a dilation.)

Proof of Lemma 3. Let $S_{1}$ be the original square. We call a square in the horizontal plane "standard" if it has the same center as $S_{1}$ and has sides parallel to those of $S_{1}$. Assign the sides of $S_{1}$ length 2 . Let $S_{0}$ and $S_{2}$ be standard squares with sides of length 3 and 1 respectively (see figure $1 \mathrm{~b}$ ). Define $h$ to be the identity on and outside $S_{1}$. Define $h$ on $S_{2}$ and its interior by the linear map taking the corners of $S_{2}$ to the corresponding corners of the top surface of the cylinder. Note that for $x$ and $y$ on or inside $S_{2}, d(h(x), h(y))=2 d(x, y)$.

Suppose $S$ is a standard square inside the annular region bounded by $S_{1}$ and $S_{2}$. Let $t$ be the distance from $S$ to $S_{1}$. Then we let $h$ map $S$ to the horizontal square in the cylinder at a height of $f(t)=2 C \sqrt{t}$ from the plane. The corners of $S$ are mapped to the corresponding corners of the image square and the sides are then mapped linearly. Choose $C$ so that $C \sqrt{2}$ equals the height of the cylinder: $C \geqq 2 \sqrt{2}$. It's easy to see $h$ is a homeomorphism, so it remains to show that $h$ is a local dilation.

First we make some remarks. If $x$ and $y$ lie on the same standard square then $h(x)$ and $h(y)$ are at least as far apart as $x$ and $y$. Also, $f^{\prime}(t)=C / \sqrt{t}$. Now consider a segment of length $a$, lying in the annular region bounded by $S_{1}$ and $S_{2}$, and lying on a line through the center of $S_{1}$ (a radial segment). Then $h$ maps the segment to a vertical segment. Since the endpoints of the radial segment are on squares a distance of at least $a / \sqrt{2}$ apart (say at distances $s$ and $t$ from $S_{1}$ ), the image segment has length $a^{\prime}=|f(t)-f(s)|=f^{\prime}(w)|t-s| \geqq$ $f^{\prime}(w) a / \sqrt{2}$ for some $w$ between $s$ and $t$. For $t \geqq w, a^{\prime} \geqq C a / \sqrt{2 w} \geqq$ $2 a / \sqrt{t}$, and we see the image segment has length $a^{\prime} \geqq 2 a$. We generally will use the prime (') symbol to indicate image points or image lengths. For $x$ and $y$ in the domain of $h$, we let $d=\operatorname{dist}(x, y)$ and $d^{\prime}=$ $\operatorname{dist}(h(x), h(y))$.

Now we show that $h$ is a local dilation in the annular region bounded by $S_{1}$ and $S_{2}$. There are two cases to consider. First assume $x$ and $y$ are in the same one of the four trapezoidal subregions formed by the diagonals of $S_{1}$ (see the lower part of figure $2 \mathrm{a}$ ). We allow $x$ or $y$ to lie on the boundary of this region, but can assume $s<t$, where $y(x)$ is on the standard square $S_{s}\left(S_{t}\right)$ which is $s(t)$ units from $S_{1}$. Construct the radial segment from $x$ to $S_{s}$, call its length $a$. Let $b(\geqq 0)$ 
PROJECTIONS IN $E^{3}$

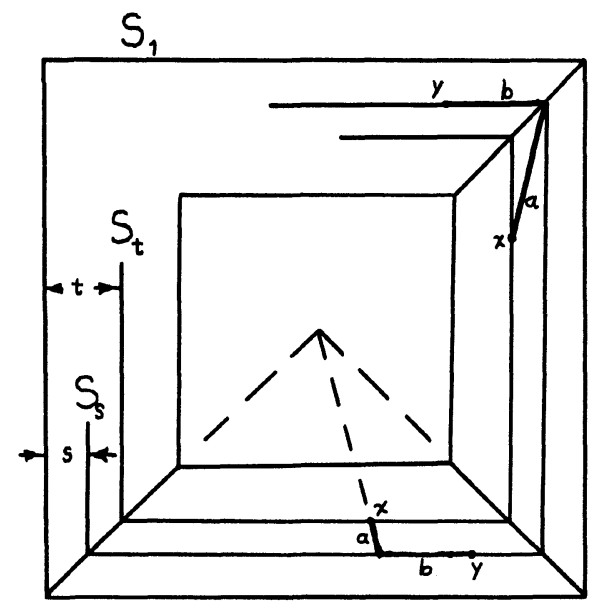

Figure 2a

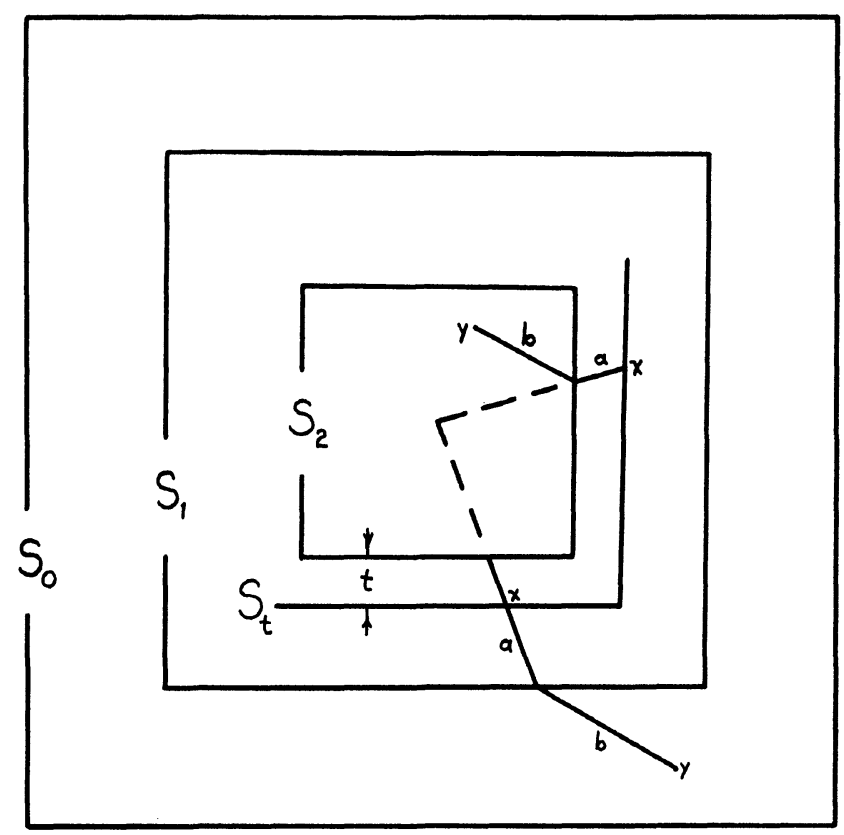

Figure 2b 
be the length of the segment in $S_{s}$ completing the path from $x$ to $y$ (refer again to the lower part of figure 2a). Facts at our disposal include: $t-s \leqq a, 0 \leqq s<t \leqq 1 / 2, \quad 1 /(1-s)^{2} \geqq 1+2 s$ (a true inequality for $s<1), \quad a^{\prime} \geqq 2 a / \sqrt{t} \geqq a, \quad b^{\prime}=b /(1-s) \geqq b$. We can assume $b \leqq 1$ (if $b>1$, then $d^{2} \leqq a^{2}+b^{2} \leqq\left(a^{\prime}\right)^{2}+\left(b^{\prime}\right)^{2}=$ $\left.\left(d^{\prime}\right)^{2}\right)$. Also $d^{2} \leqq a^{2}+b^{2}+a b \sqrt{2}$ (law of cosines), $\left(d^{\prime}\right)^{2}=\left(a^{\prime}\right)^{2}+$ $\left(b^{\prime}\right)^{2}$. If $t \leqq 2 a / b$, then $\left(d^{\prime}\right)^{2} \geqq\left(4 a^{2} / t\right)+b^{2}=\left(a^{2} / 2 t\right)+b^{2}+\left(7 a^{2} / 2 t\right)$ $\geqq a^{2}+b^{2}+(7 a b / 4) \geqq d^{2}$. If $t \geqq 2 a / b$, then $s \geqq t-a \geqq(2 a-a b) / b$ $\geqq a / b$, and $\left(d^{\prime}\right)^{2} \geqq a^{2}+\left(b^{2} /(1-s)^{2}\right) \geqq a^{2}+b^{2}+2 s b^{2} \geqq a^{2}+b^{2}$ $+2 a b \geqq d^{2}$. (If $b=0, d^{\prime}=a^{\prime} \geqq a=d$ ). Hence $\left(d^{\prime}\right)^{2} \geqq d^{2}$.

Now assume $x$ and $y$ are in adjacent trapezoidal regions. As in the upper part of figure 2a, let $x$ be in the smaller standard square, $a$ be the length of the segment from $x$ to the corner of $S_{s}$ shared by both trapezoids, and $b$ be the length of the segment from the corner to $y$. We already have shown that $a^{\prime} \geqq a, b^{\prime} \geqq b$, and since the angle between the segments of length $a$ and $b$ is less than $\pi / 2$, we have $d^{2} \leqq a^{2}+b^{2}$ $\leqq\left(a^{\prime}\right)^{2}+\left(b^{\prime}\right)^{2}=\left(d^{\prime}\right)^{2}$. (Note: if $a$ is the length of the segment from $x$ to $z, a^{\prime}=d\left(x^{\prime}, z^{\prime}\right)$. Hence we don't have to concern ourselves with whether the images of such segments are segments.)

It remains to look at neighborhoods of points of $S_{1}$ and $S_{2}$. We use neighborhoods of radius $1 / 2$. We can assume $x$ ( $\in S_{t}$ as before) is in the annular region bounded by $S_{1}$ and $S_{2}$, and $y$ is outside that region. Let $a$ be the length of the radial segment from $x$ to $S_{i}(i=1$ or 2 , whichever square separates $x$ from $y$ ), and $b$ the length of the segment from there to $y$ (see figure $2 \mathrm{~b}$ ). So (since $b \leqq 2$ here) $d^{2} \leqq a^{2}+b^{2}+$ $2 a b \leqq a^{2}+b^{2}+4 a$. If we are in a neighborhood of $S_{1}, b^{\prime}=b$, and since $t \geqq a / \sqrt{2},\left(a^{\prime}\right)^{2}=f(t)^{2}=4 C^{2} t \geqq 8 a \geqq 7 a+a^{2}(a \leqq 1)$. So $\left(d^{\prime}\right)^{2}=\left(a^{\prime}\right)^{2}+\left(b^{\prime}\right)^{2} \geqq a^{2}+b^{2}+7 a \geqq d^{2}$. On the other hand, if we are in a neighborhood of $S_{2}$, we have $b^{\prime}=2 b, a^{\prime} \geqq 2 a$ (by an earlier remark), so $\left(d^{\prime}\right)^{2} \geqq a^{2}+b^{2}+3\left(a^{2}+b^{2}\right) \geqq a^{2}+b^{2}+6 a b \geqq d^{2}$. Note that these calculations hold even if one of the points is on $S_{i}$ $(i=1$ or 2$)$. The proof is complete.

\section{Proof of theorem. We repeat:}

THEOREM. In $E^{3}$ there exists a tame 2-sphere with a Cantor subset such that all projections of the two sets are the same.

Proof. (a) Let $\Delta_{0,1}$ be a 3-simplex and $S_{0} \subset$ Int $\Delta_{0,1}$ a P.L. 2-sphere.

(b) By induction on $n=0,1,2, \cdots$ (steps $c$-h) we define polyhedral 3-cells $\left\{\Delta^{\prime \prime}{ }_{n, i}\right\}$ (and auxiliary 3-cells $\left\{\Delta_{n, i}\right\},\left\{\Delta^{\prime}{ }_{n, i}\right\}$ ), a P.L. 2-sphere $S_{n}$, a homeomorphism $h_{n}: S_{n-1} \rightarrow S_{n}$, and $\epsilon_{n}>0$ such that: 
(i) $S_{n}$ meets each Int $\Delta^{\prime \prime}{ }_{n, i}$.

(ii) For some $\delta(n)>0: h_{n}(x) \neq x$ only if $x \in\left(\Delta^{\prime \prime}{ }_{n-1, i}\right)^{\delta}$ for some $i$, and then $h_{n}(x) \in\left(\Delta^{\prime \prime}{ }_{n-1, i}\right)^{\delta}$.

(iii) $h_{n}$ is a (uniform) local dilation (i.e., there exists $\gamma(n)>0$ such that $d(x, y)<\gamma$ implies $\left.d(x, y) \leqq d\left(h_{n}(x), h_{n}(y)\right)\right)$.

(iv) For some $\beta(n)>0$, any line meeting $S_{n}$ meets $\left(\cup_{i} \Delta^{\prime \prime}{ }_{n, i}\right)^{\beta}$.

(v) diam $\Delta^{\prime \prime}{ }_{n, i}<\epsilon_{n},\left\{\Delta^{\prime \prime}{ }_{n, i}\right\}$ are pairwise disjoint.

Furthermore, we will make sure that for $n>0$,

(vi) $\Delta^{\prime \prime}{ }_{n, i} \subset \Delta^{\prime \prime}{ }_{n-1, j}$ for some $j ; \quad \Delta^{\prime \prime}{ }_{n-1, j}$ contains at least two $\Delta^{\prime \prime}{ }_{n, i} ;$ and $d\left(h_{n-1}(x), h_{n-1}(y)\right)<d(x, y)$ implies $d\left(h_{n-1}(x), h_{n-1}(y)\right)>$ $2 \epsilon_{n}$.

(c) Fix $n>0$ and assume the above for $n-1$ (i-v hold for $n=0$ trivally, if we define $S_{-1}=S_{0}, h_{0}=i d, \Delta^{\prime \prime}{ }_{0,1}=\Delta_{0,1}$, etc.). Choose $0<\epsilon_{n}<1 / 2^{n}$ small enough so that $\epsilon_{n}<\min _{i} \operatorname{diam}\left(\Delta^{\prime \prime}{ }_{n-1, i}\right)$ and so that if $d\left(h_{n-1}(x), h_{n-1}(y)\right)<d(x, y)$ then $d\left(h_{n-1}(x), h_{n-1}(y)\right)>2 \epsilon_{n}$. This latter requirement can be fulfilled as follows: by iii there is a $\gamma>0$ such that $d\left(h_{n-1}(x), h_{n-1}(y)\right)<d(x, y)$ implies $d(x, y)>\gamma$. Cover $S_{n-1}$ by finitely many compact $\gamma / 3$ neighborhoods $N_{1}, \cdots, N_{k}$. If $d\left(h_{n-1}(x), h_{n-1}(y)\right)<d(x, y)$, then $x$ and $y$ are in disjoint $N_{i}$ and $N_{j}$. But $h_{n-1}$ is a homeomorphism, so $h_{n-1}(x)$ and $h_{n-1}(y)$ are in disjoint $h_{n-1}\left(N_{i}\right)$ and $h_{n-1}\left(N_{j}\right)$. Let $2 \epsilon_{n}<\min \left(\operatorname{dist}\left(h_{n-1} N_{i}, h_{n-1} N_{j}\right): N_{i}\right.$ and $N_{j}$ are disjoint).

(d) Triangulate $\cup_{i} \Delta^{\prime \prime}{ }_{n-1, i}$ into $\left\{\Delta_{n, i}\right\}$, with mesh less than $\epsilon_{n}$ and in general position. Note that since $\left\{\Delta^{\prime \prime}{ }_{n-1, i}\right\}$ are pairwise disjoint, this amounts to triangulating each $\Delta^{\prime \prime}{ }_{n-1}, i$ separately. The general position used here is with respect to $S_{n-1}$ inside each $\Delta^{\prime \prime}{ }_{n-1, i}$. Specifically, $\quad \partial \Delta_{n, i} \cap S_{n-1} \cap \operatorname{Int} \cup \Delta^{\prime \prime}{ }_{n-1, j}$ is empty or 1-dimensional, and vertices of $\Delta_{n, i}$ inside Int $U \Delta^{\prime \prime}{ }_{n-1, j}$ are not in $S_{n-1}$. This can be accomplished in the usual method of first taking a small triangulation, and then adjusting vertices in Int $\Delta^{\prime \prime}{ }_{n-1, j}$ as necessary (still a triangulation by $[6$, p. 370]).

(e) Within each $\Delta^{\prime \prime}{ }_{n-1, i}$ we wish to "push out" $S_{n-1}$ to meet each $\Delta_{n, j}$ in $\Delta^{\prime \prime}{ }_{n-1, i}$. We proceed by constructing $\left\{p_{m}\right\}$, a finite collection of finite polygonal trees sprouting from $S_{n-1}$ into $\left\{\right.$ Int $\left.\Delta_{n, j}\right\} .\left\{p_{m}\right\}$ is constructed by taking for the start of each $p_{m}$ polygonal arcs from $S_{n-1}$ which branch off into those $\Delta_{n, j}$ which don't meet $S_{n-1}$ but are adjacent (by a 2 -face) to a $\Delta_{n, j}$ which meets $S_{n-1}$. Then we consider $\Delta_{n, k}$ adjacent to those and so on. This process is used so that we can insure that $p=\bigcup p_{m}$ meets $\Delta_{n, i}$ in a single connected set (in fact a polygonal tree) for those $\Delta_{n, i}$ which don't meet $S_{n-1}$. Further, it's easy to see from our construction that we can take: 
(i) $\left\{p_{m}\right\}$ pairwise disjoint.

(ii) $p \subset$ Int $\Delta^{\prime \prime}{ }_{n-1, i}$.

(iii) $p$ misses the 1-skeleton of $\left\{\Delta_{n, i}\right\}$.

(iv) The "vertices" of $p$ lie in $\bigcup$ Int $\Delta_{n, i}$. $S_{n-1}$.

(v) $p_{m} \cap S_{n-1}=$ a single point lying in the interior of a 2 -face of

(vi) The segment of $p_{m}$ meeting $S_{n-1}$ is perpendicular to that face.

(vii) Any two segments of $p_{m}$ which meet are perpendicular, and each vertex of $p_{m}$ lies in at most two segments of $p_{m}$ (perhaps in the interior of one of the segments).

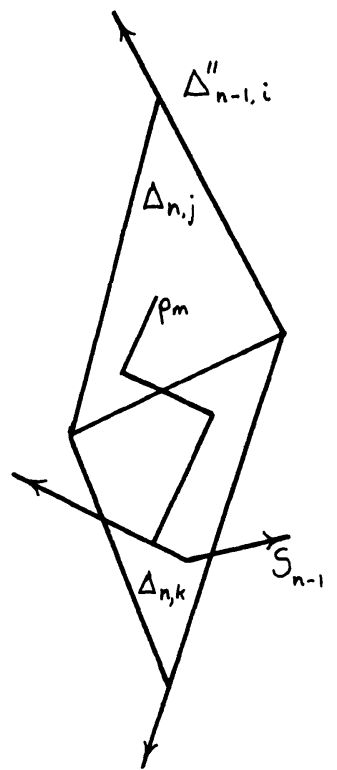

(a)

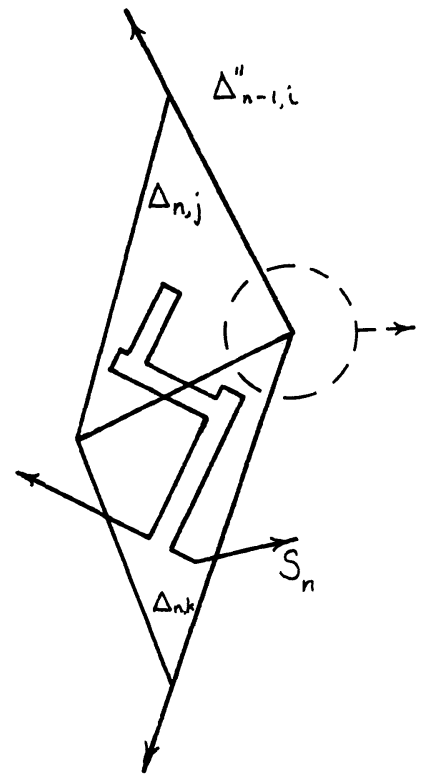

(b)

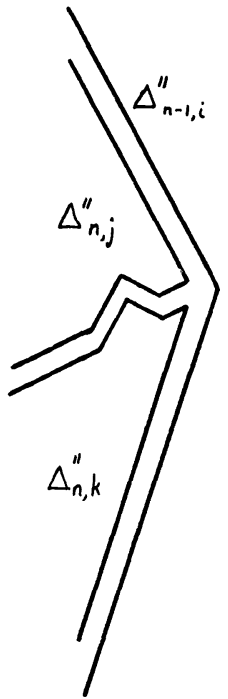

(c)

Figure 3

(See the 2-dimensional schematic in figure 3a.)

(f) The construction in (e) makes it easy to "push out" $S_{n-1}-a$ small square patch around $p_{m} \cap S_{n-1}$ is pushed out so as to closely drape $p_{m}$. More precisely, by Lemma 3, a small square patch in $S_{n-1}$ can be mapped to the sides and top of a cylinder which approximates the segment of $p_{m}$ meeting $S_{n-1}$. The map is a local dilation of $S_{n-1}$ (it is the identity outside the patch). Proceeding inductively on the rest of $p_{m}$ we see that after a finite number of steps we have defined 
$h_{n}$, a homeomorphism and local dilation with domain $S_{n-1}$, so that $S_{n}=h_{n}\left(S_{n-1}\right)$ approximates $S_{n-1} \cup \cup p_{m}$ as close as we want. We can, in fact, insure that the set of points moved by $h_{n}$, and the image under $h_{n}$ of this set, are some positive distance from $\partial \Delta^{\prime \prime}{ }_{n-1, i}$. It follows that $h_{n}$ satisfies (b ii). Since $S_{n}$ meets each Int $\Delta_{n, j}$ in $\Delta^{\prime \prime}{ }_{n-1, i}$, we have "almost" satisfied (b i). And $h_{n}$ satisfies (b iii) since a composition of continuous local dilations is a local dilation (uniformity holds since the domain of $h_{n}$ is compact). $h_{n}$ is actually the last stage of an ambient isotopy (restricted to $S_{n-1}$ ) which moves points within a compact subset of $\bigcup$ Int $\Delta^{\prime \prime}{ }_{n-1, i}$ (this will be proven later). Note further that if $x \neq h_{n}(x)$ and $h_{n}$ moves $x$ from outside $\Delta_{n, j}$ into $\Delta_{n, j}$, then $S_{n} \cap \Delta_{n, j} \subset h_{n}\left(\Delta_{n, i} \cap S_{n-1}\right)$ for some $i$.

(g) By (b iv), any line meeting $S_{n-1}$ meets $\left(\cup_{i} \Delta^{\prime \prime}{ }_{n-1, i}\right)^{\beta}$ for some $\beta>0$. Since $h_{n}: S_{n-1} \rightarrow S_{n}$ only moves points within a compact subset of $\bigcup$ Int $\Delta^{\prime \prime}{ }_{n-1, i}$, any line meeting $S_{n}$ meets $\left(\bigcup_{i} \Delta^{\prime \prime}{ }_{n-1, i}\right)^{\mu}$ for some $\boldsymbol{\mu}>0$. So by Lemma 1 , we can alter $\Delta_{n, i}$ arbitrarily slightly to $\Delta^{\prime}{ }_{n, i}$ (as in the lemma), another subdivision, so that any line meeting $S_{n}$ meets $U\left(\Delta^{\prime}{ }_{n, i}\right)^{\gamma}$ for some $\gamma>0$ and $\operatorname{diam}\left(\Delta^{\prime}{ }_{n, i}\right)<\epsilon_{n}$. In $\Delta_{n, i} \rightarrow$ $\Delta^{\prime}{ }_{n, i}$ we can make sure points move by less than $\epsilon_{n} / 4$. Further, by Lemma 1 , since $\left\{\Delta_{n, i}\right\}$ are in general position with respect to the cylindrical neighborhoods of $p(=K$ in the lemma $)$, we can make sure that for $x \neq h_{n}(x), h_{n}(x) \in \Delta_{n, i}$ iff $h_{n}(x) \in \Delta^{\prime}{ }_{n, i}$.

(h) "Shrink" each $\Delta^{\prime}{ }_{n, i}$ slightly to $\Delta^{\prime \prime}{ }_{n, i}$ so that $\left(\Delta^{\prime}{ }_{n, i}\right)^{\gamma} \subset$ Int $\Delta^{\prime \prime}{ }_{n, i}$ $\subset \Delta^{\prime \prime}{ }_{n, i} \subset$ Int $\Delta^{\prime}{ }_{n, i}$ and $S_{n}$ still meets each $\Delta^{\prime \prime}{ }_{n, i}$ with general position (e.g., $\Delta^{\prime}{ }_{n, i}$ is P.L. homeomorphic to the standard 3-cell, so we just take a slightly smaller 3-cell). The $\left\{\Delta^{\prime \prime}{ }_{n, i}\right\}$ are 3-cells and we can thus satisfy (b i-v). Only left is (vi), but this was satisfied in (c) by our choice of $\epsilon_{n}$. In $\Delta_{n, i} \rightarrow \Delta^{\prime}{ }_{n, i} \rightarrow \Delta^{\prime \prime}{ }_{n, i}$ we can make sure points move by less than $\epsilon_{n} / 2$.

(i) We have now satisfied (b). Let $C=\bigcap_{n} \bigcup_{i} \Delta^{\prime \prime}{ }_{n, i}$, a tame Cantor set by the preliminary remarks. Here we use the fact that each $\Delta^{\prime \prime}{ }_{n-1, j}$ contains at least two $\Delta^{\prime \prime}{ }_{n, i}$. Let $\Sigma=\bigcup_{n}\left(S_{n}-\bigcup_{i} \Delta^{\prime \prime}{ }_{n, i}\right)$ and $S=\Sigma \cup C$.

(j) $S$ is a 2-sphere: $h_{n}: S_{n-1} \rightarrow E^{3}$. By (b ii), $d\left(x, h_{n}(x)\right)<$ $\max \operatorname{diam}\left(\Delta^{\prime \prime}{ }_{n-1, i}\right)<\epsilon_{n-1}<1 / 2^{n-1} \quad\left(\right.$ or $<\epsilon_{0}$ for $n=1$ ), so by Lemma $2, h_{n} \circ h_{n-1} \circ \cdots \circ h_{1} \rightarrow h$ a continuous function.

Also by Lemma $2, R(h)=S$ : By (b ii), $h_{n+1}$ is fixed on points of $S_{n}$ in $\sim \bigcup_{i} \Delta^{\prime \prime}{ }_{n, i}$. So $S_{n+1} \supset S_{n}-\bigcup_{i} \Delta^{\prime \prime}{ }_{n, i}$. Since $\bigcup_{i} \Delta^{\prime \prime}{ }_{n, i}$ decreases in $n$ (by (b vi), $S_{n+1}-\bigcup \Delta^{\prime \prime}{ }_{n+1, i} \supset S_{n}-\bigcup \Delta^{\prime \prime}{ }_{n, i}$. So $\Sigma=\bigcup_{n}\left(S_{n}-\right.$ $\left.\cup \Delta^{\prime \prime}{ }_{n, i}\right)$ is an increasing union. If $x \in \Sigma$, then $x \in S_{n}$ for all $n$ greater than some $N$. If $x \in C$, then for $1 / 2^{n}<\epsilon, \epsilon_{n}<\epsilon$ and $x \in \Delta^{\prime \prime}{ }_{n, i}$ for some $i$, but $S_{n}$ meets $\Delta^{\prime \prime}{ }_{n, i}$, so $\operatorname{dist}\left(x, R\left(h_{n-1}\right)\right)<\epsilon_{n}<\epsilon$. Thus by the 
lemma, $\Sigma \cup C \subset R(h)$. If $x \notin \Sigma \cap C$, then $x \notin \cup \Delta^{\prime \prime}{ }_{n, i}$ for some $n$, and so $x \notin S_{n} \cup \cup \Delta^{\prime \prime}{ }_{n, i}$. But for $m>n, S_{m} \subset S_{n} \cup \cup_{i} \Delta^{\prime \prime}{ }_{n, i}$ (iterating (b ii) and (b vi) and so $x$ is a positive distance from $R\left(h_{m}\right)$. Hence $x \notin R(h)$. So $S=\Sigma \cup C=R(h)$.

Therefore $h: S_{0} \rightarrow S$ is a continuous surjection. To complete $(\mathrm{j})$ we show $h$ is $1-1$. Suppose $x \neq y, h(x)=h(y)$. Then for some $n>0$, $d\left(h_{n}\left(x^{\prime}\right), h_{n}\left(y^{\prime}\right)\right)<d\left(x^{\prime}, y^{\prime}\right)$ where $x^{\prime}=h_{n-1} \circ \cdots \circ h_{1}(x)$ and similarly for $y^{\prime}$. By (b vi), $d\left(h_{n}\left(x^{\prime}\right), h_{n}\left(y^{\prime}\right)\right)>2 \epsilon_{n+1}$ and so $h_{n}\left(x^{\prime}\right)$ and $h_{n}\left(y^{\prime}\right)$ are in different $\Delta_{n+1, i}$. But $h(x)=h(y)$ implies $h_{n+1}\left(h_{n}\left(x^{\prime}\right)\right)$ and $h_{n+1}\left(h_{n}\left(y^{\prime}\right)\right)$ are in the same $\Delta^{\prime \prime}{ }_{n+1, i}$ (otherwise we can find an $r>0$ such that for $m>n, h_{m} \circ \cdots \circ h_{1}(x)$ and $h_{m} \circ \cdots \circ h_{1}(y)$ are greater than $r$ apart). In $\Delta_{n+1, i} \rightarrow \Delta^{\prime \prime}{ }_{n+1, i}$ points move by less than $\epsilon_{n+1} / 2$. But $\operatorname{diam}\left(\Delta^{\prime \prime}{ }_{n+1, i}\right)<\epsilon_{n+1}, d\left(h_{n}\left(x^{\prime}\right), h_{n}\left(y^{\prime}\right)\right)>2 \epsilon_{n+1}$. So we must have that $h_{n+1}$ moves one of the points (say $h_{n}\left(x^{\prime}\right)$ ) into $\Delta^{\prime \prime}{ }_{n+1, i}$ from outside $\Delta^{\prime \prime}{ }_{n+1, i}$. Then (by construction in $f$ and $g$ ) $S_{n+1} \cap \Delta^{\prime \prime}{ }_{n+1, i}$ consists of points pushed into $\Delta_{n+1, i}$ from some single $\Delta_{n+1, j}$. So $h_{n}\left(x^{\prime}\right)$ and $h_{n}\left(y^{\prime}\right)$ are in $\Delta_{n+1, j}$, a contradiction.

Hence $h: \mathrm{S}_{0} \rightarrow \mathrm{S}$ is a homeomorphism.

(k) If a line meets $S$, it meets $C$, because: $1 \cap S \neq \varnothing$ implies $1 \cap$ $C \neq \varnothing$ or $1 \cap\left(S_{n}-\cup_{i} \Delta^{\prime \prime}{ }_{n, i}\right) \neq \varnothing$ for some $n$. We assume the latter. But by $(\mathrm{j}), S_{m} \supset S_{n}-\bigcup \Delta^{\prime \prime}{ }_{n, i}$ for all $m \geqq n$. So 1 meets $\bigcup \Delta^{\prime \prime}{ }_{m, i}$ for all $m \geqq n$ by (b iv). $\left\{\bigcup_{i} \Delta^{\prime \prime}{ }_{m, i}\right\}$ are compact and decrease to $C$, so by the usual argument, 1 meets $C$.

(l) We have now proven that there exists a tame Cantor set on a 2 -sphere with the projection property of the theorem. It remains to show that the 2-sphere can be taken tame. In effect we redo the same construction with two modifications: 1 . the previous construction used arbitrary triangulation (of small enough mesh) and we will now be more specific in our choice of triangulation, 2 . we will be more careful in our choice of the segments forming $\left\{p_{m}\right\}$.

(m) Let $U_{m}=\left\{y \in E^{3}: \operatorname{dist}\left(y, S_{0}\right)>1 / m\right\}$. Going back to (e) and (f) we see that $h_{n}$ was the last stage of an ambient isotopy restricted to $S_{n-1}$ (Pushing a plane region over a right cylinder as was done in (e) can be extended to an isotopy on $E^{3}$ which is fixed outside a neighborhood of the solid cylinder. Just take a slightly larger cylinder and extend linearly "as necessary" from a point just below the center of the original base square.). Call the last stage of this isotopy $H_{n}$. Since $h_{n}$ only "moves" points inside a compact subset of $\bigcup \Delta^{\prime \prime}{ }_{n-1, i}$, we can insure that:

(1) $H_{n}$ is the identity outside a compact subset of $\bigcup \Delta^{\prime \prime}{ }_{n-1, i}$.

(2) $H_{n}\left(\Delta^{\prime \prime}{ }_{n-1, i}\right) \subset \Delta^{\prime \prime}{ }_{n-1, i}$.

(3) $\left.H_{n}\right|_{s_{n-1}}=h_{n}$. 
Also since $\Delta_{n, i} \rightarrow \Delta^{\prime}{ }_{n, i}$ was taken to move points away from the cylindrical neighborhoods of $p_{m}$, we can assure $H_{n}$ is fixed on points moved by $\Delta_{n, i} \rightarrow \Delta^{\prime}{ }_{n, i}$.

(n) We will construct a homeomorphism $H^{\prime}{ }_{n}$ with properties 2 and 3 of $H_{n}$ and: $\left(1^{\prime}\right) H^{\prime}{ }_{n}$ is the identity outside $\bigcup \Delta^{\prime \prime}{ }_{n-1, i}$. Also, we will have $H^{\prime}{ }_{n} \circ H^{\prime}{ }_{n-1} \circ \cdots \circ H^{\prime}{ }_{1}\left(U_{n}\right) \subset \sim \cup \Delta^{\prime \prime}{ }_{n, j}$. Now $U^{\prime}{ }_{n}=H^{\prime}{ }_{n-1}$ 。 $\cdots \circ H^{\prime}{ }_{1}\left(U_{n}\right)$ is a positive distance from $S_{n}$. We will define $H^{\prime}{ }_{n}=$ $H_{n} \circ F^{\prime} \circ f$, where $F^{\prime} \circ f$ pushes $U^{\prime}{ }_{n}$ into a part of $\sim \cup \Delta^{\prime \prime}{ }_{n, j}$ which is unaffected by $H_{n}$.

(o) $f$ will be fixed on $S_{n}$ and outside $\cup \Delta^{\prime \prime}{ }_{n, i}$, so it suffices to look at one component of $\Delta^{\prime \prime}{ }_{n, i}-S_{n}$. Let $R$ be the closure of one such component. $\quad \partial R=\left(R \cap S_{n}\right) \cup\left(R \cap \partial \Delta^{\prime \prime}{ }_{n, i}\right)$, neither of these two sets being empty. Remember $S_{n}$ is in general position with respect to $\Delta^{\prime \prime}{ }_{n, i}$. Let $A_{1}, \cdots, A_{k}$ be the components of $A=R \cap \partial \Delta^{\prime \prime}{ }_{n, i}$. We show there exists $K \subset R-S_{n}$, a (at most) 2-dimensional polyhedron with the following property: given $\delta>0$, there exists (the last stage of) an isotopy $g: R \rightarrow R, g$ fixed on $\partial R$, and for $x \in R, d\left(x, S_{n}\right)>\delta$ we have $d(g(x), K \cup A)<\delta$.

(p) To get the $K$ desired above, it suffices to show that for some triangulation we can collapse $R$ to $K \cup A$ (rel $K \cup A$ ), with $K$ as desired. This is because the collapse can be used to define an isotopy i.e., for $N$ a neighborhood of $S_{n}$, each collapse "pulls" $N \cap \Delta_{n, i}$ along a little, so that in the end $N \cap \Delta{ }_{n, i}$ fills up all of $R$ outside a neighborhood of $K \cup A$. In particular, we can use $\delta$-neighborhoods so that $g$ is as desired.

Hence it suffices to show we can collapse a triangulation of $R$ as desired. Let $M$ be a triangulation of $R$ containing a subdivision of $A(\subset \partial R)$ as a subcomplex. It's clear that we can collapse $M$ to some 2-dimensional $K^{*} \cup A^{*}\left(A^{*}\right.$ the triangulation of $A$, collapse rel $K^{*} \cup$ $\left.A^{*}\right)$ since each point of Int $R$ is accessible by a general position polygonal arc from $\partial R-A$. Hence if we were unable to collapse all 3simplices such an arc could hit some first uncollapsed 3-simplex on a face, and we could collapse it. The difficulty is that we want $K^{*} \subset$ $R-S_{n}$.

But with the correct subdivision $M^{*}$ of $M$, for $N=$ \{simplices of $M$ "meeting" $\partial R-A\}$ we can geometrically collapse $M^{*}$ to $M^{*}-N$. Namely, for $\Sigma=N \cap \dot{M}$, we let $M^{*}=S_{\Sigma}{ }^{2} M$. This is defined and proven to work by Glaser [5, pp. 70-76] in his discussion of Whitehead's regular neighborhood theorem. Especially note Glaser's remark on p. 74 that for such a subdivision one can collapse a combinatorial $n$-manifold away from the boundary. Our result is completely analogous. And so we can, with the proper triangulation, collapse 
$R$ to $K \cup A$ (rel $K \cup A$ ), with $K \subset$ Int $R$ and $K$ 2-dimensional ( $K=$ $\left.\left|K^{*}\right|, A=\left|A^{*}\right|\right)$.

Thus $g$ is defined on each such $R$, and $f$ is hence defined as the union of these $g$ 's, and the identity outside $\bigcup \Delta^{\prime \prime}{ }_{n, i}$.

(q) $F$ is the ambient isotopy $\Delta_{n, j} \rightarrow \Delta_{n, j}^{\prime}$. We summarize our final construction:

1. Assume we've constructed $S_{n-1},\left\{\Delta^{\prime \prime}{ }_{n-1, i}\right\}$.

2. Construct the 2-dimensional polyhedron $K(=K(n-1))$. The closure of each component of $\Delta^{\prime \prime}{ }_{n-1, i}-S_{n-1}$ collapses into $K \cup$ $\partial \Delta^{\prime \prime}{ }_{n-1, i}$ in the component.

3. Triangulate each $\Delta^{\prime \prime}{ }_{n-1, i}$ into $\left\{\Delta_{n, j}\right\}$ with mesh less than $\epsilon_{n}$ and less than $d\left(K, S_{n-1}\right) / 3$, and in general position with respect to $S_{n-1}$ (as described in $d$ ). But further, we can assume a subdivision of $K$ is contained as a subcomplex (this does not conflict with general position since $K$ is a positive distance from $S_{n-1}$ ).

4. The collapse of each component of $\Delta^{\prime \prime}{ }_{n-1, i}-S_{n-1}$ into part of $K \cup \partial \Delta^{\prime \prime}{ }_{n-1, i}$ shows we can define $\left\{p_{m}\right\}$ from $S_{n-1}$ to any point of Int $\Delta^{\prime \prime}{ }_{n-1, i}-K$; in particular to each Int $\Delta_{n, j}$ with $p_{m}$ disjoint from $K$ and missing the edges of $\left\{\Delta_{n, j}\right\}$ (and $\left\{p_{m}\right\}$ still fulfill the conditions of $(e))$.

5. Define $h_{n}: S_{n-1} \rightarrow S_{n}$ using $\left\{p_{m}\right\}$ as in $(f)$, but not affecting any point of $K, \partial \Delta^{\prime \prime}{ }_{n-1, i}$ or the edges of $\left\{\Delta_{n, j}\right\}$. Define the natural extension $H_{n}$, fixed on some neighborhood of $K$ and the edges of $\left\{\Delta_{n, j}\right\}$, and outside $\bigcup \Delta^{\prime \prime}{ }_{n-1, i,}$.

6. Define $\Delta_{n, j} \rightarrow \Delta^{\prime}{ }_{n, j}$ as before, by an ambient isotopy $F$. By Lemma 1, we can assume $F$ doesn't move points of $K$ outside the above (in 5) mentioned neighborhood of $K$, so that $H_{n}$ is fixed on $F(K)$. Consider the collection of vertices of those $\Delta_{n, j}$ which meet $K$. The shift $E \rightarrow E^{\prime}$ restricted to this set defines an isotopy $F^{\prime}$, by leaving all other vertices fixed and extending linearly (We implicitly assume here that the original shift was sufficiently small so that any such "subshift" would define an isotopy). By (q) 3 above, $F^{\prime}$ is fixed on $S_{n-1}$. Let $K^{\prime}=F^{\prime}(K)=F(K)$.

7. Define $\Delta^{\prime}{ }_{n, j} \rightarrow \Delta^{\prime \prime}{ }_{n, j}$, a homeomorphism shrinking each $\Delta^{\prime}{ }_{n, j}$. Hence $K^{\prime \prime} \subset 2$ 2-skeleton of $\left\{\Delta^{\prime}{ }_{n, j}\right\}$, is outside each $\Delta^{\prime \prime}{ }_{n, j}$.

8. Let $U^{\prime}{ }_{n}=H^{\prime}{ }_{n-1} \circ \cdots \circ H^{\prime}{ }_{1}\left(U_{n}\right), \sim U^{\prime}{ }_{n}$ is a neighborhood of $S_{n-1}$.

9. The collapse of each component of $\Delta^{\prime \prime}{ }_{n-1, i}-S_{n-1}$ into $K \cup$ $\partial \Delta^{\prime \prime}{ }_{n-1, i}$ shows how to define $f: f$ pushes $\sim U^{\prime}{ }_{n}$ out to engulf all of $\Delta^{\prime \prime}{ }_{n-1, i}$ except a small neighborhood of $K \cup \partial \Delta^{\prime \prime}{ }_{n-1, i}$ (Note that $f$ is a homeomorphism fixed on $S_{n-1} \cup \partial \Delta^{\prime \prime}{ }_{n-1, i}$ ). $F$ and $F^{\prime}$ make this small neighborhood a neighborhood of $K^{\prime \prime} \cup \partial \Delta^{\prime \prime}{ }_{n-1, i}$. We choose 
$f$ to make the neighborhood small enough so that for $H^{\prime}{ }_{n}=H_{n}$ 。 $F^{\prime} \circ f, H^{\prime}{ }_{n}\left(U^{\prime}{ }_{n}\right) \cap \Delta^{\prime \prime}{ }_{n-1, i}$ is very close to $K^{\prime \prime} \cup \partial \Delta^{\prime \prime}{ }_{n-1, i}$, namely $H^{\prime}{ }_{n} \circ H^{\prime}{ }_{n-1} \circ \cdots \circ H^{\prime}{ }_{1}\left(U_{n}\right)$ is outside $U \Delta^{\prime \prime}{ }_{n, j} \quad\left(H_{n}\right.$ is fixed on $\left.K^{\prime \prime} \cup \partial \Delta^{\prime \prime}{ }_{n-1, i}\right)$.

10. Note that $f, F^{\prime}, H_{n}$, and hence $H^{\prime}{ }_{n}$ are fixed outside $\cup \Delta^{\prime \prime}{ }_{n-1, i}$. Since $f$ and $F^{\prime}$ are each the identity on $S_{n-1}$, we have $\left.H^{\prime}{ }_{n}\right|_{S_{n-1}}=$ $\left.H_{n}\right|_{S_{n-1}}=h_{n}$.

(r) $H^{\prime}{ }_{n}$ only moves points within each $\Delta^{\prime \prime}{ }_{n-1, i}$, so it moves points by less than $\epsilon_{n-1}<1 / 2^{n-1}$. Hence by Lemma $2, H^{\prime}{ }_{n} \circ H^{\prime}{ }_{n-1} \circ \cdots \circ H^{\prime}{ }_{1}$ converges to a continuous function $H$. Since $H$ is the identity outside $\Delta_{0,1}, R(H)=E^{3}$ and $H$ is a homeomorphism if it is 1-1. Suppose $H(x)=H(y)$.

Case 1: $x, y \in S_{0}$. Then $h(x)=H(x)=H(y)=h(y)$. By $(\mathrm{j}) h$ is $1-1$, so $x=y$.

Case 2: $x, y \notin S_{0}$. Then there exists $n$ such that $x, y \in U_{n} . H^{\prime}{ }_{n}^{\circ}$ $\cdots \circ H^{\prime}{ }_{1}\left(U_{n}\right) \subset \sim \cup \Delta^{\prime \prime}{ }_{n, j}$. For $m>n, \quad H^{\prime}{ }_{m}$ is fixed outside $\bigcup \Delta^{\prime \prime}{ }_{m-1, i} \subset \bigcup \Delta^{\prime \prime}{ }_{n, j}$. So $H$ is $1-1$ on $U_{n}$ and $x=y$.

Case 3: $x \in S_{0}, y \notin S_{0}$ (and vice versa). Then $y \in U_{n}$ for some $n$. But as in case 2, $H(y) \notin \cup \Delta^{\prime \prime}{ }_{n, j} \cup S_{n}$. But $H(x) \in S \subset \cup \Delta^{\prime \prime}{ }_{n, j}$ $\cap S_{n}$. So this case leads to a contradiction.

(s) Hence $H$ is a homeomorphism of $E^{3}, H\left(\mathrm{~S}_{0}\right)=h\left(\mathrm{~S}_{0}\right)=\mathrm{S}$. In fact, $H$ is the last stage of an isotopy. So $S$ is tame. II

Further Corollaries:

Corollary 5. We can construct $\mathrm{S}$ as in the theorem but with the unit ball inside $S$ and the exterior of the $(1+\epsilon)$-ball containing $S$. In fact, starting with $S_{0} \subset$ Int $P, P$ any 3-dimensional polyhedron, we can find $S$ as in the theorem so that $S \subset P$ and for $x \notin P, x$ is inside $S$ iff $x$ is inside $S_{0}$.

Proof. Just start with $\left\{\Delta_{0, i}\right\}$ a triangulation of $P$. The above construction then does the job.

Corollary 6. The theorem provides an alternate construction of a result due to Bing ([3, Theorem 6.3, p. 443]): There is a countable collection of tame Cantor sets in $E^{3}$ such that each straight line interval in $E^{3}$ intersects one of these Cantor sets.

Proof. By Corollary 5, there is a sequence $S_{i}$ of spheres with the property of the theorem such that the origin is inside each $S_{i}$ and diam $S_{i} \rightarrow 0$. Consider translations of $E^{3}$ which take the origin to elements of a countable dense set. Any line interval will meet at least one of the translated $S_{i}$ 's in the same non-empty set as the line containing the interval. Hence the interval will meet the corresponding Cantor set. 


\section{Conollary 7. The theorem holds in $E^{2}$.}

Proof. The construction can be so adapted. But there is an easier construction. Let $C \subset[0,1]$ be the Cantor set formed by "deleting middle tenths". Then any line at an angle greater than $\tan ^{-1}(1 / 10)$ with the horizontal and vertical and meeting $R^{\prime}$ also meets $C^{\prime}=$ $C \times C \subset R^{\prime}=[0,1] \times[0,1]$. Rotate the plane $45^{\circ}$ about $(1 / 2,1 / 2)$ with function $r$. Then we check that $C^{\prime} \cup r\left(C^{\prime}\right)$ is a Cantor set with the same projections as $R^{\prime} \cup r\left(R^{\prime}\right)$. We can thread $C^{\prime} \cup r\left(C^{\prime}\right)$ with a simple closed curve inside $R^{\prime} \cup r\left(R^{\prime}\right)$ using two methods due to Antoine. It suffices to show that for any Cantor set, $K$, lying in a square, $R^{\prime}(=[0,1] \times[0,1])$, there is a simple closed curve in $R^{\prime}$ containing $K$. $K$ can be covered by a finite collection of arbitrarily small disjoint polygons and their interiors (lying in the plane $[4, \mathrm{p}$. $298]$ ). We can assume the polygons lie in $R^{\prime}$ by intersecting them with $R^{\prime}$. If any of these polygons separate $R^{\prime}$, we can break them up into smaller disjoint polygons which don't separate $R^{\prime}$, by a theorem of Antoine's ([4, p. 296]). Now these new polygons can be connected by polygonal arcs in $R^{\prime}$ as a "first approximation" of a simple closed curve, e.g., by first using segments of $\partial R^{\prime}$ to cyclically connect those polygons meeting $\partial R^{\prime}$ (leaving the first and last unconnected to one another) and then using arcs in Int $R^{\prime}$ to connect the other polygons in the appropriate fashion. Each polygon and interior is homeomorphic to a square and interior, and a similar method is used inductively. In the limit we get a simple closed curve (similar to [4, p. 302-4]) as desired.

\section{REFERENCES}

1. J. W. Alexander, On the Subdivision of 3-Space by a Polyhedron, Proceedings of the National Academy of Science, X (1924), 6-8.

2. Louis Antoine, Sur l'homeomorphie de deux figures et de leurs voisinages, Journal de Mathematique, IV series 8 (1921), 221-325.

3. R. H. Bing, Tame Cantor Sets in $E^{3}$, Pacific Journal of Mathematics, XI (1961), 435-446.

4. Karol Borsuk, An Example of a Simple Arc in Space whose Projection in Every Plane has Interior Points, Fund. Math., XXXIV (1947), 272-77.

5. Leslie Glaser, Geometric Combinatorial Topology, vol. I. (Van Nostrand Reinhold Mathematical Studies, no. 27) New York: Van Nostrand Reinhold Co., 1970.

6. Hassler Whitney, Geometric Integration Theory. (Princeton Mathematical Series, v. 21) Princeton: Princeton University Press, 1957.

Stanford University, Stanford, California 94305 